\title{
Disruption of the Aspergillus fumigatus ECM33 homologue results in rapid conidial germination, antifungal resistance and hypervirulence
}

\begin{abstract}
Correspondence
Nir Osherov

nosherov@post.tau.ac.il
\end{abstract}

Received 19 February 2006

Revised 20 March 2006

Accepted 22 March 2006

\author{
Jacob Romano, ${ }^{1}$ Guy Nimrod, ${ }^{2}$ Nir Ben-Tal, ${ }^{2}$ Yona Shadkchan, ${ }^{1}$ \\ Koti Baruch, ${ }^{1}$ Haim Sharon ${ }^{1}$ and Nir Osherov ${ }^{1}$
}

\begin{abstract}
Department of Human Microbiology, Sackler School of Medicine ${ }^{1}$ and Department of Biochemistry², George S. Wise Faculty of Life Sciences, Tel-Aviv University, Ramat-Aviv 69978, Tel-Aviv, Israel
\end{abstract}

\begin{abstract}
The ECM33/SPS2 family of glycosylphosphatidylinositol-anchored proteins plays an important role in maintaining fungal cell wall integrity and virulence. However, the precise molecular role of these proteins is unknown. In this work, AfuEcm33, the gene encoding the ECM33 homologue in the important pathogenic fungus Aspergillus fumigatus, has been cloned and its function analysed. It is shown that disruption of $A f u E c m 33$ results in rapid conidial germination, increased cell-cell adhesion, resistance to the antifungal agent caspofungin and increased virulence in an immunocompromised mouse model for disseminated aspergillosis. These results suggest that the protein encoded by $A f u E c m 33$ is involved in key aspects of cell wall morphogenesis and plays an important role in $A$. fumigatus virulence.
\end{abstract}

\section{INTRODUCTION}

Aspergillus fumigatus is the most common opportunistic mould pathogen of humans, causing invasive diseases in immunocompromised patients (Latgé, 1999). Invasive pulmonary aspergillosis is caused by inhalation of $A$. fumigatus spores and growth of the fungus inside the lungs, which often spreads from the initial site of infection to attack various organs in the body. It is the leading cause of death in leukaemia and bone-marrow-transplant patients, with between 10 and $20 \%$ of patients contracting this disease. Left untreated, mortality rates from this disease are extremely high $(>90 \%)$, and even following aggressive antifungal treatment, fatality rates of 50 to $70 \%$ are common (Steinbach \& Stevens, 2003). Therefore, there is an urgent need for a deeper understanding at the molecular level of the interaction of $A$. fumigatus with the infected host.

The fungal cell wall plays a crucial role in infection. In $A$. fumigatus, as in other pathogenic fungi, the cell wall is in continuous contact with the host, acting as a reservoir for displayed and secreted antigens and enzymes. The cell wall protects the fungus and interacts directly with the host immune system. It is an elastic, dynamic and highly regulated structure, and is essential for growth, viability and infection. The cell wall of A. fumigatus is composed of a

\footnotetext{
Abbreviations: CWP, cell wall protein; DDW, double-distilled water; GPI, glycosylphosphatidylinositol.

Figures showing sequence alignments and a phylogenetic tree are available as supplementary data with the online version of this paper.
}

polysaccharide skeleton interlaced and coated with cell wall proteins (CWPs). The main building blocks of the polysaccharide skeleton are an interconnected network of glucan, chitin and galactomannan polymers (Latgé et al., 2005). The cell wall of A. fumigatus differs considerably from that of yeast such as Saccharomyces cerevisiae and Candida albicans. It contains a much higher level of chitin and unique polysaccharides such as 1,3 - $\alpha$-glucan, $(1,3)(1,4)$ - $\beta$-glucan and galactomannan (Latgé et al., 2005).

The major class of fungal CWPs is the glycophosphatidylinositol (GPI)-modified proteins (de Groot et al., 2003; Eisenhaber et al., 2004). They contain an N-terminal hydrophobic signal peptide sequence that targets them to the endoplasmic reticulum (ER), and a C-terminal hydrophobic domain that is cleaved off in the ER and replaced with a GPI anchor. The GPI anchor directs the attachment of these proteins to the plasma membrane. Subsequently, the GPI anchor may be processed and attached to $1,6-\beta$ glucan in the cell wall. ECM33/SPS2-family proteins have the typical features of GPI-anchored proteins, with a signal peptide, a serine and threonine-rich region and a potential C-terminal domain for GPI-anchor attachment (PercivalSmith \& Segall, 1987; Caro et al., 1997; Tougan et al., 2002; Terashima et al., 2003). They play an important role in fungal cell wall organization. Deletion of S. cerevisiae ECM33 results in a weakened and disorganized cell wall, defects in glycosylation, and activation of the cell wall integrity pathway (Pardo et al., 2004). The C. albicans $\mathrm{CaEcm} 33$ protein is required for normal cell wall architecture and expression of cell-surface proteins. $\mathrm{CaEcm} 33$-deleted mutants display a 
reduced ability to invade and damage epithelial cells, and show decreased virulence in a murine model of systemic candidosis (Martinez-Lopez et al., 2004, 2006).

Proteomic analysis of $A$. fumigatus membrane preparations identified nine GPI-anchored proteins, including the protein encoded by AfuEcm33 (Afu4g06820), the homologue of S. cerevisiae ECM33 (Bruneau et al., 2001). In light of the importance of the ECM33/SPS2-family proteins in cell wall organization and virulence, we have undertaken the characterization of the A. fumigatus ECM33 homologue, AfuEcm33 (Afu4g06820). This is believed to be the first time that such a characterization has been undertaken in a pathogenic filamentous fungus. Interestingly, we show that disruption of $A f u E c m 33$ in A. fumigatus results in rapid conidial germination, increased cell-cell adhesion, resistance to the antifungal caspofungin and increased virulence in an immunocompromised mouse model for disseminated aspergillosis.

\section{METHODS}

Strains and culture conditions. A. fumigatus strain AF293, originally isolated at autopsy from a patient with invasive pulmonary aspergillosis, and A. fumigatus strain AF293.1, a derivative of the AF293 strain (Pain et al., 2004), were used throughout this study. The AF293.1 strain is deficient in pyrG (encoding OMP-decarboxylase) and, consequently, is auxotrophic for uridine/uracil (Osherov et al., 2001). AF293.1 was grown on YAG UU medium, which consists of $0.5 \%(\mathrm{w} / \mathrm{v})$ yeast extract, $1 \%(\mathrm{w} / \mathrm{v})$ glucose, $10 \mathrm{mM} \mathrm{MgCl} 2,10 \mathrm{mM}$ uracil and $5 \mathrm{mM}$ uridine, supplemented with trace elements, vitamins and $1.5 \%(\mathrm{w} / \mathrm{v})$ agar when needed (Bainbridge, 1971). For phenotypic analysis, mutants were grown on minimal medium (MM) containing $70 \mathrm{mM} \mathrm{NaNO}, 1 \%$ glucose, $12 \mathrm{mM}$ potassium phosphate $\mathrm{pH} 6 \cdot 8,4 \mathrm{mM} \mathrm{MgSO}, 7 \mathrm{mM} \mathrm{KCl}$, trace elements and $1.5 \%$ agar (for MM agar plates). Conidia were harvested in $0.2 \%$ $(\mathrm{v} / \mathrm{v})$ Tween 80 , resuspended in double-distilled water (DDW) and counted with a haemocytometer. Escherichia coli strain DH10B (Invitrogen) was used to clone and replicate the genes in the pGEMT/A and pGPS1/pyr4 transposon-containing vectors (Jadoun et al., 2004).

RNA analysis. AF293 and disrupted strains AfuEcm33-D1-4 were grown for the indicated time in liquid $\mathrm{MM}$ at $37^{\circ} \mathrm{C}$. Total RNA was extracted by the 'hot SDS' protocol (May \& Morris, 1988). Northern blot analysis was performed as described previously (Osherov et al., 2002). Briefly, $5 \mu \mathrm{g}$ fungal total RNA was run on a $1 \%(\mathrm{w} / \mathrm{v})$ agarose gel under denaturing conditions, transferred to a Nytran $\mathrm{N}$ nylon membrane (Schleicher \& Schuell) and hybridized with an $\left[\alpha-{ }^{32} \mathrm{P}\right] \mathrm{dCTP}$ radiolabelled $A f u E c m 33$ full-length probe at $47^{\circ} \mathrm{C}$. For RT-PCR, total RNA was treated with DNase (Ambion) according to the manufacturer's instructions. The RNA concentration was assessed and $3 \mu \mathrm{g}$ were taken for the RT reaction using PowerScript reverse transcriptase (Clontech). PCR was performed using ReddyMix PCR master mix (ABgene) with the following designed primer pairs (Table 1): AfuEcm33 forward and reverse primers were used to identify the AfuEcm 33 transcript, and AfugpdA forward and reverse primers were used as a loading control. The PCR was carried out according to the manufacturer's instructions. PCR products were analysed by gel electrophoresis.

Construction and verification of the A. fumigatus AfuEcm33 disruption mutant. Disruption of $A f u E c m 33$ was performed using an in vitro transposon-based mutagenesis approach as described previously (Jadoun et al., 2004). Briefly, this approach utilizes a modified transposon containing the Neurospora crassa pyr4 gene, which is randomly inserted in vitro into a target sequence of interest. Clones in which the gene of interest has been disrupted are identified by PCR and used to transform a pyrG-deficient strain of $A$. fumigatus. Primers were designed to contain an AscI restriction site at their $5^{\prime}$ end (Table 1) and generated a $\sim 4 \mathrm{~kb}$ fragment using the Expand high-fidelity PCR system (Roche Diagnostics). The primer pairs used to amplify the fragments for the $A f u E c m 33$ gene were $A f u E c m 33+A s c I$ site forward and $A f u E c m 33+A s c I$ site reverse (Table 1). A. fumigatus AF293 $\mathrm{CsCl}_{2}$-purified genomic DNA (1 $\mu \mathrm{g}$ per reaction) was used as a template in the reactions. PCR conditions were as recommended by the manufacturer. The PCR fragment produced was gel purified by the Wizard SV gel and clean-up system (Promega), and cloned into the pGEM T/A cloning vector (Promega), according to the manufacturer's instructions, to obtain pGEM-AfuEcm33. Inactivation of the AfuEcm33 gene was performed using the GPS-1 genome priming system (New England Biolabs) with the modified GPS-1/pyr4 transposon (Jadoun et al., 2004) according to the manufacturer's instructions. Clones carrying transposon-disrupted genes were identified by PCR using AfuEcm 33 forward and reverse primers (Table 1). Sequencing indicated that the transposon inserted 624 bp downstream of the AfuEcm 33 gene start codon. The gene fragment with the transprimer 1-pyr4 disruption, and its flanking sequence, was released by cleavage with AscI, purified with the Wizard SV gel and clean-up system and used to transform A. fumigatus strain AF293.1 (Jadoun et al., 2004). Rapid initial screening for positive disrupted mutants was performed by PCR amplification of crude conidial genomic DNA. Conidia from transformed colonies were collected and transferred to $500 \mu \mathrm{l}$ DDW to give a conidial concentration of $\sim 10^{7}$ conidia $\mathrm{ml}^{-1}$. The tubes were snap frozen in liquid nitrogen for $10 \mathrm{~min}$, heated at $95^{\circ} \mathrm{C}$ for $5 \mathrm{~min}$ and used for PCR with the AfuEcm33 forward and reverse primers (Table 1). Amplification of the housekeeping gpdA gene was used as a positive control. Colonies negative for gene amplification

Table 1. Primers

\begin{tabular}{|c|c|c|}
\hline Name of primer & Sequence & Product length (bp) \\
\hline AfuEcm 33 forward & 5'-CCATTGAGATCGACGAGCACCT-3' & \\
\hline AfuEcm 33 reverse & 5'-CATGCTGATGTTAGTGAGCTTGCT-3' & 635 \\
\hline AfugpdA forward & 5'-TCTCCAACGTTCTTGCACC-3' & \\
\hline AfugpdA reverse & 5'-CCACTCGTTGTCGTACCAGG-3' & 600 \\
\hline$A f u E c m 33+A s c$ I site forward ${ }^{*}$ & 5'-ATGGCGCGCCCCCGTAGTCCAGGTGATGAC-3' & \\
\hline$A f u E c m 33+A s c I$ site reverse ${ }^{\star}$ & 5'-ATGGCGCGCCGACCGGTCGGTCCTTGTA-3' & 4037 \\
\hline
\end{tabular}

${ }^{\star}$ Italics indicate the AscI restriction site included in the primer sequence. 
but positive for $g p d A$ amplification were chosen for further analysis. For further Southern blot analysis, A. fumigatus genomic DNA was extracted with a MasterPure yeast DNA purification kit (Epicenter), with modifications for A. fumigatus as described by Jin et al. (2004). Southern hybridization analysis was performed as described previously (Jadoun et al., 2004). Briefly, $10 \mu \mathrm{g}$ fungal genomic DNA were digested with HindIII and run on a $1 \%(\mathrm{w} / \mathrm{v})$ agarose gel. The cleaved DNA was transferred to a Nytran $\mathrm{N}$ nylon membrane (Schleicher \& Schuell) and hybridized with an $\alpha^{32}[\mathrm{dCTP}]$ radiolabelled N. crassa pyr4 probe at $65^{\circ} \mathrm{C}$. The AF293.1 pyr4 complemented strain was obtained by transforming the auxotrophic AF293.1 strain with the $N$. crassa pyr4 gene. The AfuEcm33 KI strain was prepared by complementing the AfuEcm33-D1 strain with plasmid pGEM-AfuEcm33 containing the pTrpC-hyg cassette (Punt et al., 1987). Three AfuEcm33 KI strains were obtained and verified for AfuEcm 33 mRNA expression by RT-PCR. All three strains were phenotypically identical to the control wild-type strain AF293 as assessed by clumping, aerosolization and germination experiments (data not shown).

\section{Phenotypic analysis of the AfuEcm33 disrupted mutant}

Growth assay. A. fumigatus AF293 and disrupted AfuEcm33 mutant isolates were grown at a concentration of $10^{4}$ conidia $\mathrm{ml}^{-1}$ in $\mathrm{MM}$ without glucose supplemented with $1 \%(\mathrm{w} / \mathrm{v})$ raffinose, sucrose, glycerol, ethanol or BSA as sole carbon sources. For growth experiments at $37^{\circ} \mathrm{C}$ and $42^{\circ} \mathrm{C}$, MM containing $1 \%$ glucose (w/v) was used. For growth analysis at different $\mathrm{pH}$ values, conidia were grown in MM buffered to $\mathrm{pH} 6$ and $\mathrm{pH} 9$ with $50 \mathrm{mM}$ BIS or citrate buffers, respectively. For analysis of growth at reduced osmo-

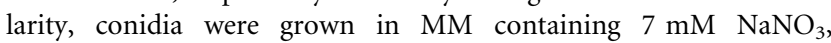
$0.4 \mathrm{mM} \mathrm{MgSO}_{4}$ and $0.7 \mathrm{mM} \mathrm{KCl}$. For growth experiments in a high osmolarity environment, $\mathrm{MM}$ containing $1.0 \mathrm{M} \mathrm{NaCl}$ was used.

Qualitative assay for conidial clumping and aerosolization. For assessment of conidial clumping, freshly harvested A. fumigatus AF293 and disrupted AfuEcm 33 mutant conidia grown for 3 days on YAG agar plates were harvested in $0 \cdot 2 \%(\mathrm{v} / \mathrm{v})$ Tween 80 , resuspended in DDW and counted with a haemocytometer. A total of $2 \times 10^{8}$ conidia $\mathrm{ml}^{-1}$ were transferred to a sterile glass tube, vortexed for $30 \mathrm{~s}$, allowed to stand for $15 \mathrm{~min}$ and photographed. For qualitative assessment of conidial aerosolization, strains were grown on YAG agar plates for 3 days. During harvesting of conidia, four sterile YAG agar plates were placed uncovered around and immediately adjacent to the harvested plate. The four plates were subsequently incubated for 2 days at $37^{\circ} \mathrm{C}$ to allow growth of aerosolized conidia. The extent of colonization, correlating to the degree of conidial aerosolization during harvesting, was assessed visually.

Microscopic analysis. Conidia at a concentration of $10^{3}$ conidia $\mathrm{ml}^{-1}$ were grown in $1 \mathrm{ml}$ liquid $\mathrm{MM}$ on glass disks in a stationary 24-well plate at $37^{\circ} \mathrm{C}$. Before microscopic examination, the conidia were stained with $8 \mu \mathrm{l}$ Fluorescent Brightener $28\left(0.5 \mu \mathrm{g} \mathrm{ml}^{-1}\right)$ (Sigma-Aldrich) and observed under an Olympus IX50 fluorescent microscope at a magnification of $\times 400$. Hyphal growth rate and germination studies were performed by plating $10^{3}-10^{5}$ freshly harvested spores $\mathrm{ml}^{-1}$ onto 96 -well plates in $200 \mu$ liquid YAG at $37^{\circ} \mathrm{C}$. At various time points, growth was observed under a gridmounted Olympus CK inverted microscope at a magnification of $\times 200$. The percentage of germinated conidia $(n=200)$ was assessed, and the lengths of the germlings $(n=50)$ were measured in microns.

Sensitivity to reagents and antifungals. A. fumigatus AF293 and disrupted $A f u E c m 33$ mutant isolates were grown in 96-well plates at a concentration of $10^{4}$ conidia $\mathrm{ml}^{-1}$ in $\mathrm{MM}$ supplemented with reagents and antifungals in 96-well plates. MICs (the lowest drug concentrations to completely arrest germination and growth) were evaluated after $24 \mathrm{~h}$ incubation at $37^{\circ} \mathrm{C}$. Unless otherwise specified, all reagents were from Sigma-Aldrich. The concentration ranges of the reagents and antifungals were: Congo red $1-160 \mu \mathrm{g} \mathrm{ml}^{-1}$; caspofungin (Merck) $1-160 \mu \mathrm{g} \mathrm{ml}^{-1}$; Calcofluor white $10-320 \mu \mathrm{g} \mathrm{ml}^{-1}$; hygromycin B 5-320 $\mu \mathrm{g} \mathrm{ml}^{-1}$; amphotericin B 1-80 $\mu \mathrm{g} \mathrm{ml}^{-1}$; itraconazole $0 \cdot 25-8 \mu \mathrm{g} \mathrm{ml}^{-1}$; tunicamycin $1-80 \mu \mathrm{g} \mathrm{ml}^{-1}$; trifluoroperazine $10-160 \mu \mathrm{g} \mathrm{ml}^{-1}$.

For sensitivity testing on agar plates, conidia from the mutant and AF293 wild-type strain $\left(10^{8}\right.$ conidia $\left.\mathrm{ml}^{-1}\right)$ were point inoculated on MM plates containing either $80 \mu \mathrm{g}$ Congo red $\mathrm{ml}^{-1}$ or $40 \mu \mathrm{g}$ caspofungin $\mathrm{ml}^{-1}$ or no drug control. The strains were grown for $48 \mathrm{~h}$ at $37^{\circ} \mathrm{C}$.

Murine model for systemic aspergillosis. Six-week-old female ICR mice were injected intraperitoneally with $200 \mathrm{mg}$ cyclophosphamide $\mathrm{kg}^{-1}$ at 3 days prior to conidial infection. Mice were inoculated intravenously via the tail vein with a $2 \cdot 5 \times 10^{5}$ conidia per mouse inoculum of freshly harvested AF293 wild-type, AfuEcm33D1 disrupted, AfuEcm33 KI (complemented) or AF293.1 pyr4 (pyr4 complemented) conidia. To prolong neutropenia, additional cyclophosphamide $\left(70 \mathrm{mg} \mathrm{kg}^{-1}\right)$ was administered 2 and 5 days after infection. Systemic aspergillosis was followed up for 30 days. Statistical analysis of mouse survival was performed with GraphPad Prism 4 software (GraphPad Software). $P$ values of $<0.05$ were considered significant in this analysis. Animal studies were performed in accordance with Tel-Aviv University institutional policies.

\section{RESULTS}

\section{Primary sequence analysis of AfuEcm33p}

The AfuEcm33 gene is 1300 nucleotides in length and contains two introns (at positions $61-131 \mathrm{bp}$ and $972-$ $1029 \mathrm{bp}$ ). AfuEcm33p (gene product of Afu4g06820, TIGR assembly) is 398 amino acids in length, rich in serine/ threonine amino acid residues $(21 \%)$, and contains a predicted signal peptide sequence at its $\mathrm{N}$-terminus and a GPI-anchor sequence at its C-terminus. The $\omega$-site of AfuEcm33p is predicted to be at sequence position ASN372, with the typical consensus of two alanines $(\omega+1, \omega+2)$ and serine ( $\omega-1)$ (Eisenhaber et al., 2004). The most likely signal peptide cleavage site is predicted to be between ALA19 and ALA20. Based on multiple sequence alignment, AfuEcm 33 p has significant sequence identity to Aspergillus nidulans hypothetical protein AN4390.2 (60\% identity in 398 amino acids), Magnaporthe grisea Ecm33-like AAX07654 (36\% identity in 326 amino acids), N. crassa Sps2-like CAD70996 (32\% identity in 333 amino acids), C. albicans CaEcm 33p (31\% identity in 318 amino acids), S. cerevisiae Ecm 33 p (31 \% identity in 319 amino acids) and S. cerevisiae Sps2p ( $28 \%$ identity in 304 amino acids). A search of the A. fumigatus database yielded a single additional predicted protein sequence with a low but significant degree of similarity to AfuEcm $33 \mathrm{p}$, the gene product of Afu6g 10290 (29\% identity in 141 amino acids). Afu6g 10290 exhibits significant similarity to S. cerevisiae PstIp (31\% identity in 172 amino acids, probability value $2 \times 10^{-7}$ ), a protein secreted by regenerating protoplasts and a member of the ECM33/SPS2 family of proteins (Pardo et al., 1999). 
A complete evolutionary analysis of AfuEcm $33 p$ using 25 sequence homologues with the ConSeq web server (http:// conseq.bioinfo.tau.ac.il/) (Berezin et al., 2004) revealed a significant conservation of large amino acid blocks between the Aspergillus species, and a low conservation towards other fungal species (see the supplementary figures available with the online journal). This is characteristic of serine/ threonine-rich CWPs containing large numbers of nucleotide repeat units.

We showed by RT-PCR, using primers $A f u E c m 33$ forward and reverse (Table 1), that AfuEcm33 is not significantly expressed in dormant conidia, but is expressed throughout germination and hyphal growth (Fig. 1).

\section{Disruption of AfuEcm33 results in abnormal clumping, reduced aerosolization and rapid germination of conidia}

To investigate the effect of loss of function of the AfuEcm33 gene in A. fumigatus, a disruption plasmid (pAfuEcm33-D) was constructed by transposon mutagenesis as described in Methods (Fig. 2a). After transformation of pAfuEcm33-D into AF293.1, 20 pyrG + transformants were purified and screened by PCR for putative insertion mutants. Four putative mutants were identified and further characterized by Southern blotting (Fig. 2b). Based on this analysis, all four transformants were disrupted in the $A f u E c m 33$ gene alone (AfuEcm33-D1-4). The indication based on Northern blot analysis is that a truncated $A f u E c m 33$ mRNA is expressed in all four disrupted strains $(\sim 1.4 \mathrm{~kb}$ in size as compared to $2.5 \mathrm{~kb}$ in the wild-type, Fig. 2c). RT-PCR indicated that this mRNA contains the first $624 \mathrm{bp}$ of $A f u E c m 33$, a short portion of the transposon, but not the $676 \mathrm{bp}$ of coding sequence downstream of the transposon (our unpublished results). Although it cannot be formally ruled out that a truncated form of AfuEcm33p protein is expressed, this form would lack the C-terminal GPI-anchor motif targeting it to the cell membrane, and therefore it is highly unlikely that it would remain functional (Terashima

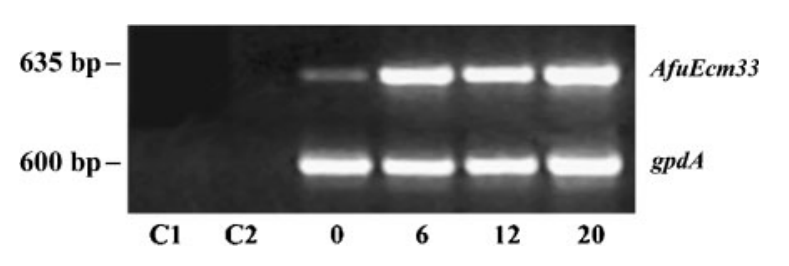

Fig. 1. Expression of the $A$. fumigatus AfuEcm33 (Afu4g06820) gene. Expression was determined by RT-PCR using primers AfuEcm33 forward and reverse (Table 1). Dormant conidia $(0 \mathrm{~h})$ were incubated for 6,12 and $20 \mathrm{~h}$ in $\mathrm{MM}$ at $37^{\circ} \mathrm{C}$, after which total RNA was isolated and used in RT-PCR. Control reactions: C1, PCR was performed on RNA following DNase treatment; C2, no DNA control. RT-PCR with primers for $g p d A$, a housekeeping gene, was performed as a control for the loading (lower bands). et al., 2003). However, to formally rule out the possibility that a phenotype is resulting from expression of a truncated form of AfuEcm $33 p$ protein, complete gene deletion mutants are being generated and their phenotype will be compared to that of the disrupted mutants described in this report.

Preliminary experiments conducted with the four independent disrupted strains confirmed that all of them exhibited the mutant phenotype, characterized by clumping, aerosolization and rapid germination. This suggests that the mutant phenotype is associated with disruption of the gene and is not due to an unlinked mutation resulting from transformation. The four strains displayed the mutant phenotype in both the absence and presence of uracil/ uridine, indicating that the mutant phenotype is not a result of positional silencing of the pyr4 marker (Greenstein et al., 2006). A representative strain, $A f u E c m 33-D 1$ was chosen for further characterization. The AfuEcm33-D1 mutant strain grew normally on rich YAG medium or defined MM agar plates, indicating that the $A f u E c m 33$ gene is not essential for growth (Fig. 3a). Freshly harvested AfuEcm33-disrupted conidia formed large aggregates in DDW, suggesting an increase in cell-cell adhesive properties (Fig. 3b). AfuEcm33-disrupted conidia showed a greatly decreased ability to aerosolize during harvesting. Sterile YAG agar plates left uncovered in the direct vicinity of harvested plates showed very high levels of colonization by conidia from wild-type plates as compared to plates containing the mutant AfuEcm33-D1 strain (Fig. 3c). Microscopic analysis of freshly harvested conidia indicated that there is no statistically significant difference in size or shape between the mutant and wild-type conidia (AF293 conidial diameter $3 \cdot 2 \pm 0 \cdot 5$ microns, AfuEcm33-D1 conidial diameter $4 \pm 0 \cdot 6$ microns, $n=100$ ). Microscopic analysis in liquid MM demonstrated that the $A f u E c m 33-D 1$ strain germinates earlier than the wild-type strain and forms large 'starshaped' clusters of germinating conidia, in which the conidial cell bodies adhere to each other and the germ tubes grow outwards, suggestive of an alteration in cell-cell adherence (Fig. 3d). The time needed for $50 \%$ of conidia to germinate was $3 \mathrm{~h} 45 \mathrm{~min}$ for the mutant AfuEcm33-D1 strain and $6 \mathrm{~h} 15 \mathrm{~min}$ for the AF293 control wild-type strain (Fig. 3e). However, the growth rates of the AF293 wild-type and mutant $A f u E c m 33-D 1$ strain were similar (compare the slopes in Fig. 3e). After 12 to $24 \mathrm{~h}$ there was no significant difference in hyphal length, colony size or morphology of the AfuEcm33-D1 strain compared to the wild-type AF293 parental strain.

\section{The AfuEcm33-D1 mutant is resistant to Congo red and caspofungin}

We tested the effects of different sole carbon sources (raffinose, sorbitol, glucose, glycerol, ethanol, casein and albumin), osmolarity, temperature $\left(24\right.$ and $\left.42^{\circ} \mathrm{C}\right), \mathrm{pH}(6 \cdot 0$ and 9.0), and various compounds and drugs, including caffeine, trifluoroperazine, hygromycin B, Calcofluor white, SDS, amphotericin B and itraconazole on the growth of the 


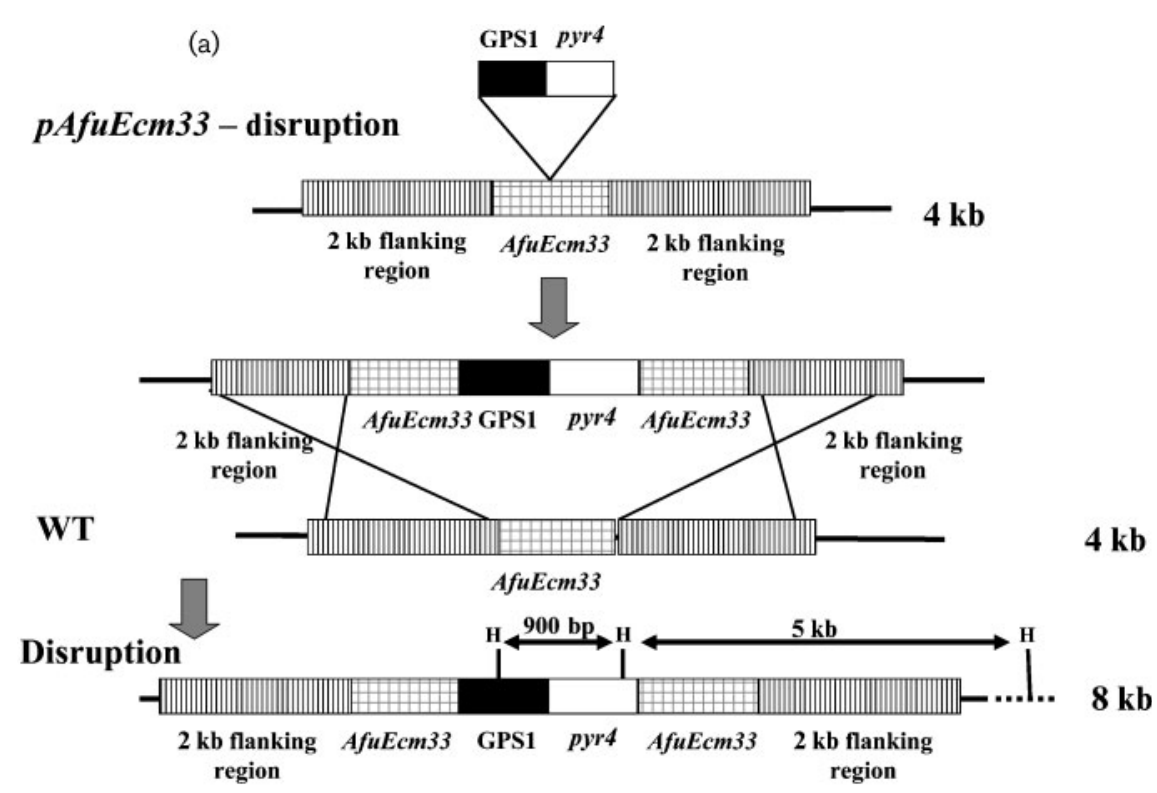

(b)

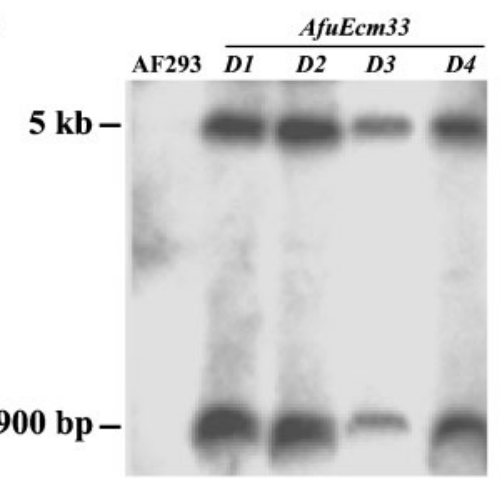

(c)

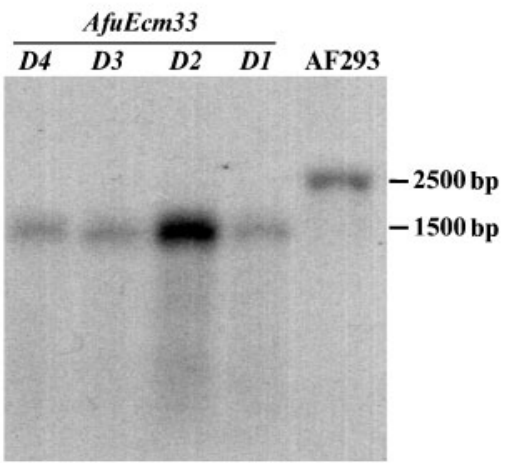

Fig. 2. Disruption of the $A f u E c m 33$ gene in A. fumigatus: (a) schematic representation of the AfuEcm33 wild-type locus and the plasmid pAfuEcm33-K/O Ascl-cut insert used for disruption, (b) Southern blot and (c) Northern blot verification of the AfuEcm33-D1-4 isolates and control AF293 wild-type strain. For the Southern blot analysis, genomic DNA (10 $\mu \mathrm{g}$ per well) was digested with Hindlll, blotted and hybridized with a ${ }^{32}$ P-labelled pyr4 DNA probe, resulting in $\sim 0.9 \mathrm{~kb}$ and $5 \mathrm{~kb}$ fragments for the AfuEcm33 disrupted strains, and no hybridization with the AF293 control strain. For the Northern blot analysis, total RNA (5 $\mu$ g per well) was hybridized with a ${ }^{32}$ P-labelled AfuEcm33 DNA probe. H, Hindlll site.

$A f u E c m 33-D 1$ strain in comparison to the wild-type AF293 strain (see Methods). The analysis was performed on MM agar plates and in liquid culture. Freshly harvested conidia were inoculated under the various conditions and grown for $24 \mathrm{~h}$ at $37^{\circ} \mathrm{C}$. We found no appreciable growth differences between the mutant and wild-type strains. In contrast, 


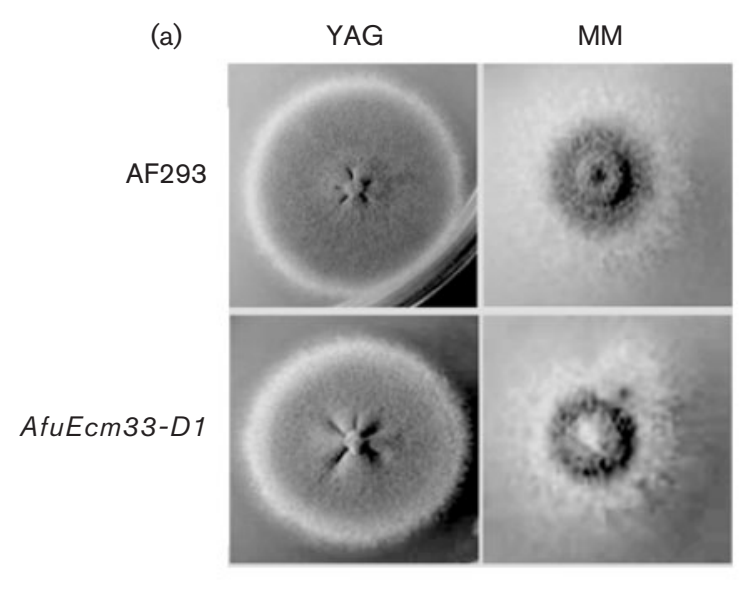

(b)

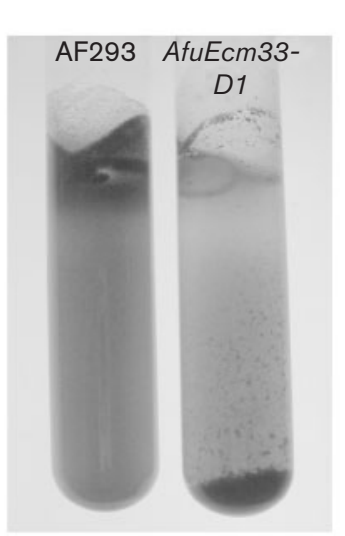

(c)
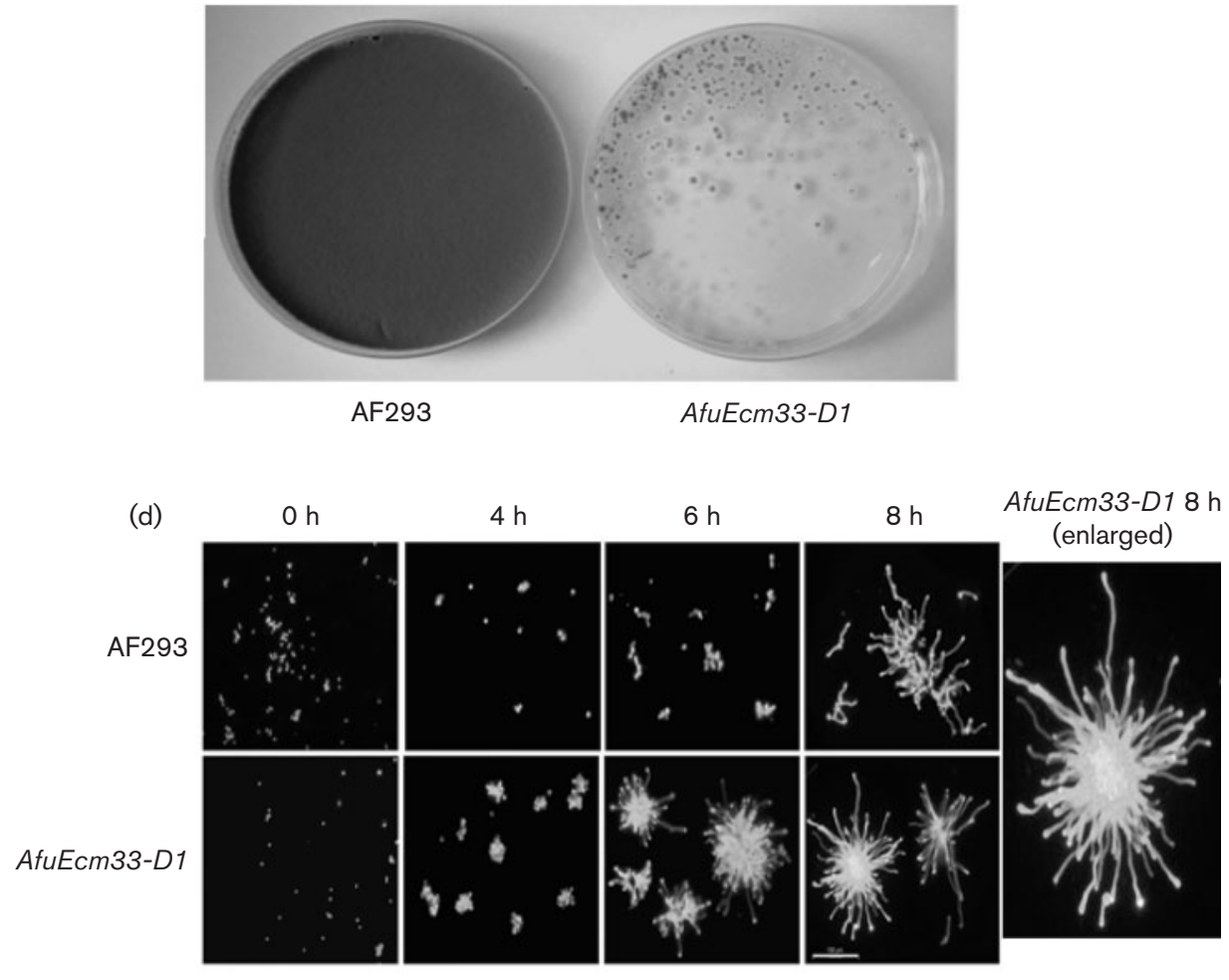

(e)
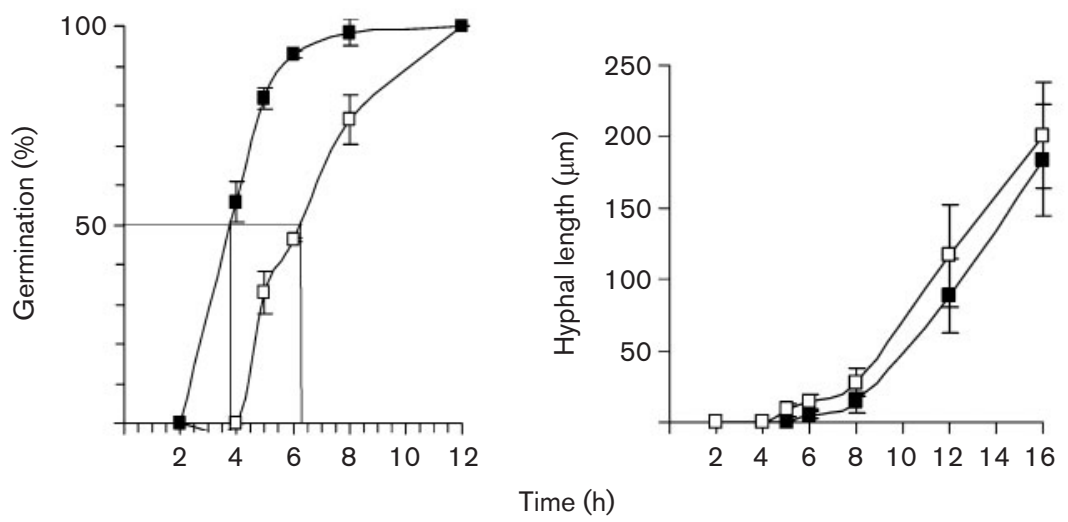


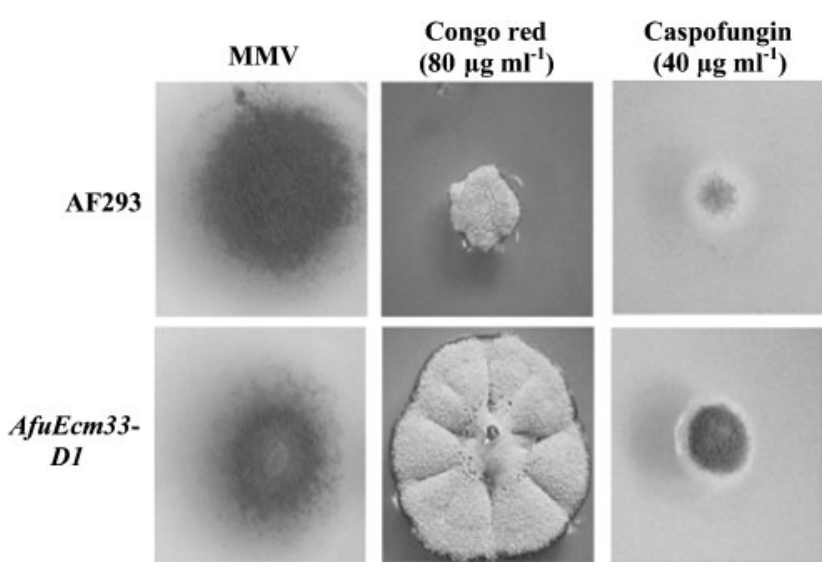

Fig. 4. The AfuEcm33-D1 mutant is resistant to the cell walldestabilizing agents Congo red and caspofungin. Conidia from the mutant and the AF293 wild-type strain were point inoculated on $\mathrm{MM}$ plates containing either $80 \mu \mathrm{g}$ Congo red $\mathrm{ml}^{-1}$ or $40 \mu \mathrm{g}$ caspofungin $\mathrm{ml}^{-1}$ or no drug (control). The strains were grown for $48 \mathrm{~h}$ at $37^{\circ} \mathrm{C}$.

growth tests conducted with the cell wall-disrupting agents Congo red or caspofungin indicated that the AfuEcm33-D1 strain is more resistant to these compounds (Fig. 4). The MIC of both agents was eightfold higher in the AfuEcm33D1 strain as compared to the AF293 control strain and was specific for these agents alone (Table 2). This result suggests that changes in the composition of the cell wall in the AfuEcm33-D1 strain may render it more resistant to damage by these agents.

\section{Disruption of AfuEcm33 increases virulence}

To determine whether the protein encoded by AfuEcm33 is involved in pathogenicity, we tested the AfuEcm33-D1 and $A f u E c m 33-D 2$ disrupted strains for virulence in an immunocompromised murine model for disseminated aspergillosis. As a control we included AF293, the parental A. fumigatus strain, AfuEcm33 KI, an AfuEcm33 complemented strain and AF293.1 pyr4, a derivative of AF293.1 that is complemented with the $N$. crassa pyr4 gene (see Methods). Freshly harvested conidia were carefully counted and adjusted to the same density $\left(2.5 \times 10^{5}\right.$ conidia per mouse) before injection into the lateral tail vein. The number of mice alive in each group was recorded every day over a 30 day study period. Fig. 5 shows survival curves
Table 2. MIC values $\left(\mu \mathrm{g} \mathrm{ml}^{-1}\right)$ for $A f u E c m 33-D 1$ and the AF293 wild-type strain incubated for $24 \mathrm{~h}$ with Congo red (CR), caspofungin (CAS), Calcofluor white (CF), itraconazole (ITZ), amphotericin B (AMB), tunicamycin (TNM) and hygromicin B (HygB)

\begin{tabular}{|lccccccc|}
\hline & CR & CAS & CF & ITZ & AMB & TNM & HygB \\
\hline AF293 & 10 & 10 & 160 & $0 \cdot 5$ & 5 & 40 & 20 \\
AfuEcm33-D1 & 80 & 80 & 80 & $0 \cdot 5$ & 5 & 40 & 40 \\
\hline
\end{tabular}

obtained during the course of the experiment. By day 7, all 14 mice injected with the AfuEcm33-D1 $(n=10)$ and AfuEcm33-D2 $(n=4)$ disrupted strains had died [mean survival time $(\mathrm{MST})=4 \cdot 1$ days]. In contrast, $60 \%$ of the mice injected with the wild-type AF293 strain or AF293.1 pyr4 strains were still alive after 30 days $(\mathrm{MST}=21$ days, $n=10$ ) while $30 \%$ of mice infected with the AfuEcm33 KI strain remained alive after 30 days (MST $=13 \cdot 2$ days, $n=10)$. Statistical analysis of these data by the Wilcoxon rank sum test showed a highly significant survival difference

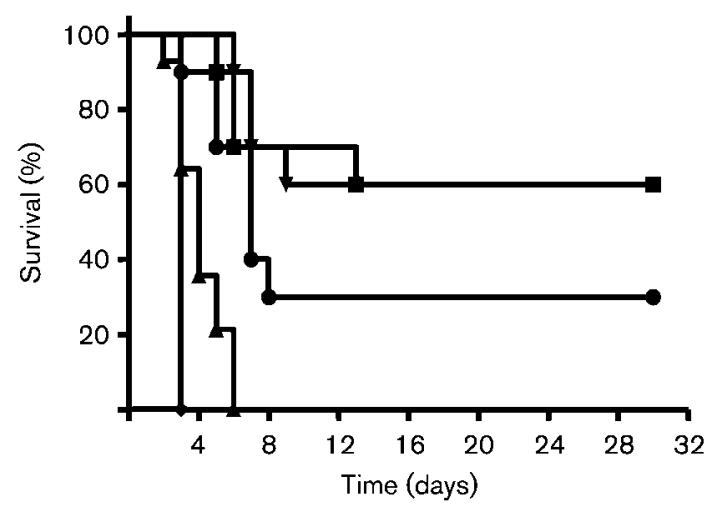

Fig. 5. The AfuEcm33 disrupted mutant shows increased virulence in a murine model of invasive aspergillosis. Survival curves are shown of mice intravenously injected with an inoculum of $2.5 \times 10^{5}$ conidia per mouse of the wild-type AF293 strain ( $\mathbf{\square})(n=10$ mice), AfuEcm33 complemented strain AfuEcm33 KI $(\bullet)(n=10)$, pyr4 complemented AF293.1 pyr4

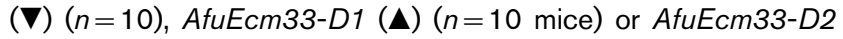
$(\boldsymbol{\nabla})(n=4$ mice) disrupted strains. Percentage survival was monitored daily over the 30 day study period.

Fig. 3. Phenotypic characterization of the $A f u E c m 33-D 1$ mutant. (a) Conidia were point inoculated on solid rich YAG and $\mathrm{MM}$ agar plates and incubated for $24 \mathrm{~h}$ at $37^{\circ} \mathrm{C}$. (b) Abnormal clumping of AfuEcm33 mutant conidia (right tube) compared to the wild-type strain (left tube) was found after harvesting and resuspending conidia in DDW. (c) Aerosolization was reduced for the AfuEcm33 mutant conidia (right plate) compared to the wild-type strain (left plate) after harvesting (see Methods). A representative result of three independent experiments is shown. (d) AfuEcm33-D1 mutant germination and early growth in liquid MM was analysed by microscope. Note the early germination and cell-cell clumping in the mutant strain (lower panel) as compared to the control AF293 wild-type strain (bar, $100 \mu \mathrm{m}$ ). (e) A quantitative analysis of percentage mutant germination and hyphal growth was carried out; each time point was calculated as the mean \pm SD of 200 conidia or 50 hyphae, respectively. $\mathbf{\square}, \mathrm{AF} 293 ; \square$, AfuEcm33-D1. 
between the mouse groups infected with the wild-type AF293, control AF293.1 pyr4, AfuEcm33 KI strains and the AfuEcm 33 disrupted A. fumigatus strains $(P<0 \cdot 0005)$, supporting a conclusion that the $A f u E c m 33$ disrupted strains exhibit hypervirulence in the tested mouse model. The survival difference between the wild-type AF293 strain and control AfuEcm33 KI strain was not statistically significant $(P=0 \cdot 19)$.

\section{DISCUSSION}

In this report, we have described the disruption of the $A$. fumigatus ECM33 homologue, AfuEcm33, and the phenotypic analysis of the mutant. We chose to study this gene because (i) deletion of S. cerevisiae ECM33 and C. albicans CaECM33 suggested that the proteins they encode play a complex but as yet poorly understood role in the maintenance of cell wall integrity, fungal morphogenesis and virulence (Martinez-Lopez et al., 2004; Pardo et al., 2004), (ii) biochemical studies had already identified AfuEcm33p as a genuine GPI-anchored protein in $A$. fumigatus (Bruneau et al., 2001) and (iii) ECM33-family proteins have not been previously characterized in the filamentous fungi.

\section{AfuEcm33 is involved in conidial adherence and morphogenesis}

Disruption of the $A f u E c m 33$ gene in A. fumigatus resulted in subtle and unusual morphological changes, including rapid germination and conidial clustering during harvesting and germination. C. albicans CaECM33-deleted cells also exhibit a marked tendency to flocculate (cluster) extensively (Martinez-Lopez et al., 2004). We hypothesize that cell wall alteration in the $A f u E c m 33$ disrupted mutant may lead to greater exposure of the cell-surface proteins involved in adherence, leading to increased cell clustering. However, the AfuEcm 33 disrupted strains exhibited no substantial differences in adherence to polystyrene (a measure of changes in cell wall hydrophobicity), laminin (a component of the extracellular matrix) or A549 lung-cell extracellular matrix as compared to the wild-type AF293 strain using either dormant or germinating conidia (our unpublished observations). This indicates that the increased cell-cell clustering we observed in the mutant is distinct from its ability to interact with the matrix.

The precocious germination of the $A f u E c m 33$ disrupted mutant is intriguing. S. cerevisiae ECM33 deleted cells exhibit marked disorganization of the cell wall, and in particular the mannoprotein outer layer (Pardo et al., 2004). Disruption of MEU10, an ECM33/SPS2-like gene in Schizosaccharomyces pombe results in the formation of fragile spores containing a weakened cell wall (Tougan et al., 2002). Perhaps disruption of $A f u E c m 33$ leads to the formation of a softer, more pliable cell wall, enabling germination to proceed more rapidly.

\section{Disruption of $A f u E c m 33$ leads to increased resistance to cell wall-disrupting agents}

Surprisingly, we found that the $A f u E c m 33$ disrupted strain is more resistant to the cell wall-disrupting agents Congo red and caspofungin (Fig. 4). This result is in contrast to that found in S. cerevisiae and C. albicans ECM33 deletion mutants, which display increased sensitivity to Congo red and Calcofluor white (Martinez-Lopez et al., 2004; Pardo et al., 2004). This could be a consequence of the considerable differences in polymer organization and cell wall content between S. cerevisiae or C. albicans and A. fumigatus (Bernard \& Latgé, 2001; Latgé et al., 2005). Resistance to the antifungal drug caspofungin typically results from mutations in its target, $(1,3)$ - $\beta$-glucan synthase (Kartsonis et al., 2003). Our work is believed to be the first to demonstrate a connection between caspofungin resistance in A. fumigatus and the loss of function of a defined gene. A plausible explanation for our finding is that disruption of $A f u E c m 33$ may lead to increased levels of chitin and $(1,3)$ - $\alpha$-glucan in the cell wall, thereby compensating for the reduced synthesis of $(1,3)$ - $\beta$-glucan by glucan synthase (Reinoso-Martin et al., 2003).

\section{The AfuEcm33 disrupted A. fumigatus mutant is hypervirulent in a murine model of disseminated aspergillosis}

We demonstrate that disruption of $A f u E c m 33$ in $A$. fumigatus leads to hypervirulence in a mouse model of disseminated aspergillosis. This is in contrast to the results obtained using the C. albicans CaECM33 disrupted mutant, which exhibited decreased virulence in a similar model system. A number of mechanisms may explain the increased virulence of the $A f u E c m 33$ mutant, including physical occlusion of blood vessels by clumps of conidia, enhanced resistance to phagocytes due to rapid germination and hyphal growth, or hyperstimulation of the immune system, leading to septic shock. Very few examples of fungal hypervirulence resulting from gene knockout have been described to date. Partial silencing of the A. fumigatus AfppoA, AfppoB and $A f p p o C$ genes encoding fatty acid dioxygenases resulted in increased resistance to oxidative stress and hypervirulence in mice (Tsitsigiannis et al., 2005). It was proposed that Ppo reaction products (prostaglandins and other oxylipins) may serve as activators of host immune defences. A decrease in prostaglandin production by the $A f p p o A-C$ silenced mutant might lead to a weaker host response resulting in hypervirulence. Disruption of the Candida glabrata ACE2 gene encoding a transcription factor and the $S$. cerevisiae SSD1 gene encoding a CWP of unknown function leads to hypervirulence in mice by inducing severe septic shock in infected animals (Wheeler et al., 2003; Kamran et al., 2004). This has been tentatively attributed to an increase in the exposure of fungal cell-surface antigens, which hyperstimulate the immune system of the infected animals (Wheeler et al., 2003). We are now performing experiments to determine whether similar mechanisms can explain the hypervirulence of the $A f u E c m 33$ disrupted mutant. 
In summary, our findings suggest that the A. fumigatus AfuEcm33 gene is involved in key aspects of cell wall architecture. The increased conidial aggregation, precocious germination, resistance to cell wall-destabilizing drugs and increased virulence resulting from the disruption of $A f u E c m 33$ suggest that significant changes in the cell wall have occurred. Further elucidation of the mechanisms responsible for these changes may shed new light on the pathogenesis of A. fumigatus at the molecular level.

\section{ACKNOWLEDGEMENTS}

We would like to thank Rotem Sorek for helpful discussions. Genomic data for A. fumigatus were provided by The Institute for Genomic Research (www.tigr.org/tdb/e2k1/aful/) and the Wellcome Trust Sanger Institute (www.sanger.ac.uk/Projects/A_fumigatus/). This work was funded by the Israel Academy of Sciences, grant 741/01 to N.O.

\section{REFERENCES}

Bainbridge, B. W. (1971). Macromolecular composition and nuclear division during spore germination in Aspergillus nidulans. J Gen Microbiol 66, 319-325.

Berezin, C., Glaser, F., Rosenberg, J., Paz, I., Pupko, T., Fariselli, P., Casadio, R. \& Ben-Tal, N. (2004). ConSeq: the identification of functionally and structurally important residues in protein sequences. Bioinformatics 20, 1322-1324.

Bernard, M. \& Latgé, J.-P. (2001). Aspergillus fumigatus cell wall: composition and biosynthesis. Med Mycol 39, 9-17.

Bruneau, J. M., Magnin, T., Tagat, E., Legrand, R., Bernard, M., Diaquin, M., Fudali, C. \& Latgé, J.-P. (2001). Proteome analysis of Aspergillus fumigatus identifies glycosylphosphatidylinositol-anchored proteins associated to the cell wall biosynthesis. Electrophoresis 22, 2812-2823.

Caro, L. H. P., Tettelin, H., Vossen, J. H., Ram, A. F. J., van den Ende, H. \& Klis, F. M. (1997). In silicio identification of glycosylphosphatidylinositol-anchored plasma-membrane and cell wall proteins of Saccharomyces cerevisiae. Yeast 13, 1477-1489.

de Groot, P. W., Hellingwerf, K. J. \& Klis, F. M. (2003). Genome-wide identification of fungal GPI proteins. Yeast 20, 781-796.

Eisenhaber, B., Schneider, G., Wildpaner, M. \& Eisenhaber, F. (2004). A sensitive predictor for potential GPI lipid modification sites in fungal protein sequences and its application to genome-wide studies for Aspergillus nidulans, Candida albicans, Neurospora crassa, Saccharomyces cerevisiae and Schizosaccharomyces pombe. J Mol Biol 337, 243-253.

Greenstein, S., Shadkchan, Y., Jadoun, J., Sharon, C., Markovich, S. \& Osherov, N. (2006). Analysis of the Aspergillus nidulans thaumatin-like cetA gene and evidence for transcriptional repression of pyr4 expression in the cetA-disrupted strain. Fungal Genet Biol 43, 42-53.

Jadoun, J., Shadkchan, Y. \& Osherov, N. (2004). Disruption of the Aspergillus fumigatus $\arg B$ gene using a novel in vitro transposonbased mutagenesis approach. Curr Genet 45, 235-241.

Jin, J., Lee, Y. K. \& Wickes, B. L. (2004). Simple chemical extraction method for DNA isolation from Aspergillus fumigatus and other Aspergillus species. J Clin Microbiol 42, 4293-4296.

Kamran, M., Calcagno, A. M., Findon, H. \& 9 other authors (2004). Inactivation of transcription factor gene $A C E 2$ in the fungal pathogen Candida glabrata results in hypervirulence. Eukaryot Cell 3, 546-552.

Kartsonis, N. A., Nielsen, J. \& Douglas, C. M. (2003). Caspofungin: the first in a new class of antifungal agents. Drug Resist Updat 6, 197-218.

Latgé, J.-P. (1999). Aspergillus fumigatus and aspergillosis. Clin Microbiol Rev 12, 310-350.

Latgé, J.-P., Mouyna, I., Tekaia, F., Beauvais, A., Debeaupuis, J. P. \& Nierman, W. (2005). Specific molecular features in the organization and biosynthesis of the cell wall of Aspergillus fumigatus. Med Mycol 43, S15-S22.

Martinez-Lopez, R., Monteoliva, L., Diez-Orejas, R., Nombela, C. \& Gil, C. (2004). The GPI-anchored protein $\mathrm{CaEcm} 33 \mathrm{p}$ is required for cell wall integrity, morphogenesis and virulence in Candida albicans. Microbiology 150, 3341-3354.

Martinez-Lopez, R., Park, H., Myers, C. L., Gil, C. \& Filler, S. G. (2006). Candida albicans Ecm33p is important for normal cell wall architecture and interactions with host cells. Eukaryot Cell 5, 140-147.

May, G. S. \& Morris, N. R. (1988). Developmental regulation of a conidiation-specific $\beta$-tubulin in Aspergillus nidulans. Dev Biol 128, 406-414.

Osherov, N., Kontoyiannis, D. P., Romans, A. \& May, G. S. (2001). Resistance to itraconazole in Aspergillus nidulans and Aspergillus fumigatus is conferred by extra copies of the A. nidulans P-450 14- $\alpha$ demethylase gene, pdmA. J Antimicrob Chemother 48, 75-81.

Osherov, N., Mathew, J., Romans, A. \& May, G. S. (2002). Identification of conidial-enriched transcripts in Aspergillus nidulans using suppression subtractive hybridization. Fungal Genet Biol 37, 197-204.

Pain, A., Woodward, J., Quail, M. A. \& 22 other authors (2004). Insight into the genome of Aspergillus fumigatus: analysis of a $922 \mathrm{~kb}$ region encompassing the nitrate assimilation gene cluster. Fungal Genet Biol 41, 443-453.

Pardo, M., Monteoliva, L., Pla, J., Sánchez, M., Gil, C. \& Nombela, C. (1999). Two-dimensional analysis of proteins secreted by Saccharomyces cerevisiae regenerating protoplasts: a novel approach to study the cell wall. Yeast 15, 459-472.

Pardo, M., Monteoliva, L., Vázquez, P., Martínez, R., Molero, G., Nombela, C. \& Gil, C. (2004). PSTI and ECM33 encode two yeast cell surface GPI proteins important for cell wall integrity. Microbiology 150, 4157-4170.

Percival-Smith, A. \& Segall, J. (1987). Increased copy number of the $5^{\prime}$ end of the SPS2 gene inhibits sporulation of Saccharomyces cerevisiae. Mol Cell Biol 7, 2484-2490.

Punt, P. J., Oliver, R. P., Dingemanse, M. A., Pouwels, P. H. \& van den Hondel, C. A. (1987). Transformation of Aspergillus based on the hygromycin B resistance marker from Escherichia coli. Gene 56, 117-124.

Reinoso-Martin, C., Schuller, C., Schuetzer-Muehlbauer, M. \& Kuchler, K. (2003). The yeast protein kinase C cell integrity pathway mediates tolerance to the antifungal drug caspofungin through activation of Slt $2 \mathrm{p}$ mitogen-activated protein kinase signaling. Eukaryot Cell 2, 1200-1210.

Steinbach, W. J. \& Stevens, D. A. (2003). Review of newer antifungal and immunomodulatory strategies for invasive aspergillosis. Clin Infect Dis 37, S157-S187.

Terashima, H., Hamada, K. \& Kitada, K. (2003). The localization change of $\mathrm{Ybr} 078 \mathrm{w} / \mathrm{Ecm} 33$, a yeast GPI-associated protein, from the plasma membrane to the cell wall, affecting the cellular function. FEMS Microbiol Lett 218, 175-180. 
Tougan, T., Chiba, Y., Kakihara, Y., Hirata, A. \& Nojima, H. (2002). Meu10 is required for spore wall maturation in Schizosaccharomyces pombe. Genes Cells 7, 217-231.

Tsitsigiannis, D. I., Bok, J.-W., Andes, D., Nielsen, K. F., Frisvad, J. C. $\&$ Keller, N. P. (2005). Aspergillus cyclooxygenase-like enzymes are associated with prostaglandin production and virulence. Infect Immun 73, 4548-4559.

Wheeler, R. T., Kupiec, M., Magnelli, P., Abeijon, C. \& Fink, G. R. (2003). A Saccharomyces cerevisiae mutant with increased virulence. Proc Natl Acad Sci U S A 100, 2766-2770. 